BARBARA KORNACKA

Università Adam Mickiewicz di Poznań

iD https://orcid.org/0000-0001-7143-404X

\title{
Memoria e storia nelle narrazioni postcoloniali di Regina di fiori e di perle di Gabriella Ghermandi e Memorie di una principessa etiope di Martha Nasibù
}

Memory and history in the postcolonial narrations of Regina di fiori e di perle by Gabriella Ghermandi and Memorie di una principessa etiope by Martha Nasibù

AвSTRACT: The aim of thise paper is to show how the setting out of the narrative voice determines the historical discourse. The analysis of the narrative voice leads to some considerations about memory and to the examination of recollection in these two novels. That, in turn, allows an exploration of the way in which the historical discourse is constructed. In those cases where the voice in the historical discourse is given to subaltern subjects, they contribute to a more plural history.

KEY wORDS: memory, history, postcolonial narrative, Gabriella Ghermandi, Martha Nasibù

\section{Impostazione teorica}

Vorrei iniziare la mia analisi da alcune osservazioni che vanno intese come un'impostazione teorica del presente contributo: una cornice paradigmatica entro la quale desidero inserire la mia riflessione sulla letteratura, ma anche, cosa inevitabile, visto l'argomento menzionato nel titolo, sulla storiografia. L'esame della letteratura postcoloniale facendo parte degli studi postcoloniali e, allargando un po' il campo di indagine, anche degli studi subalterni, rientra nel paradigma delle nuove scienze umane (new humanities), disciplina e campo di sapere 
che da alcuni decenni raduna diversi studi interdisciplinari quali ad esempio studi culturali, studi postcoloniali, gender studies, queer studies o animal studies $^{1}$. Sono studi che rappresentano atteggiamento di critica e di opposizione nei confronti del potere nonché, e soprattutto, nei confronti del sapere, delle discipline o delle istituzioni che legittimano i sistemi del potere ${ }^{2}$. I nuovi studi umani contestano quindi l'autorità del sapere canonico e promuovono i campi di ricerca finora discriminati, subalterni o non riconosciuti come studi accademici. Il loro interesse si focalizza sulla prospettiva di chi è o di chi è stato vittima, o di chi si è sempre trovato solo nella posizione dell'oggetto di ricerca (DomAŃsKA, 2006: 17-18). Come avverte la studiosa polacca Ewa Domańska, il termine stesso, new humanities, potrebbe risultare tuttavia deviante, essendo la critica delle nuove scienze umane puntata proprio contro l'umanesimo e il suo retaggio culturale e filosofico, inteso come antropocentrismo, europocentrismo, fallocentrismo $\mathrm{o}$ androcentrismo.

L'analisi delle due opere della letteratura italiana postcoloniale che si vuole proporre - La regina di fiori e di perle di Gabriella Ghermandi e Le memorie di una principessa etiope di Martha Nasibù ${ }^{3}$ - si collocherà a pieno titolo nel paradigma critico delle nuove scienze umane, non solo perché la voce narrante perlopiù appartiene alle donne di origine africana, quindi è una voce doppiamente subalterna, ma anche perché entrambi i libri parlano dell'invasione italiana, della guerra italo-etiopica (1935-1936) e della successiva colonizzazione dell'Etiopia. Entrambi quindi costituiscono una forma del discorso storico, prodotto tuttavia da parte dei soggetti vinti.

Le indagini svolte gravitano attorno a due grandi nozioni quali la memoria e la storia, in stretta correlazione tra di loro, e a loro volta, con la narrazione $e^{4}$. Quest'ultima in tali rapporti viene intesa non soltanto come un canale di trasmissione degli esiti della conoscenza acquisita, ovvero come uno strumento di sistemazione del sapere - cosa che deriva dalla riflessione sulla storia e sulla

${ }^{1}$ Da elencare vi sarebbero inoltre lesbian studies, vari studi etnici, thing studies. Cfr. DomańsKa (2006: 17).

${ }^{2}$ Sono da sottolineare in questo ambito i pionieristici lavori di critica del potere di Michele Foucault quali ad esempio i suoi corsi presso il Collège de France intitolati Il faut défendre la société, tra cui quello del 21 gennaio del 1976 in cui spiega che la disciplina storica e il discorso storico è uno strumento del potere. Foucault scrive: « L'histoire, c'est le discours du pouvoir, le discours des obligations par lesquelles le pouvoir soumet, c'est aussi le discours de l'éclat par lequel le pouvoir fascine, terrorise, immobilise ». Cfr. FouCAult (2012: 48).

${ }^{3}$ Le due opere escono a distanza di poco tempo, rispettivamente nel 2007 e nel 2005. Tutte le citazioni inserite nell'articolo provengono dalle edizioni di riferimento (GHERMANDI, 2011; NASIBÙ, 2012) e saranno contrassegnate con le sigle, rispettivamente GG e MN, nonché il numero della pagina.

${ }^{4}$ Le numerose concezioni che inquadrano il rapporto tra narrazione e storia sono studiate e riferite da Katarzyna Rosner (2006). Alla memoria e alla narrazione sono dedicate le riflessioni di Paul Ricoeur (2006). Si veda anche a tale proposito l'articolo di Mirosław Loba (2004). 


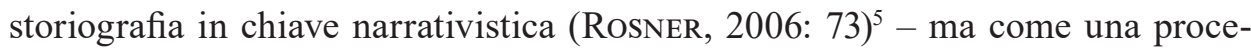
dura stessa dell'epistemologia.

Il presente articolo ha lo scopo di mostrare come attraverso l'impostazione della voce narrante si costruisce il discorso storico. Il testo dunque prevede in primo luogo, previa una presentazione delle opere esaminate, una breve analisi narratologica. In secondo luogo, si passerà a un esame della costruzione della memoria attraverso le strategie narrative. Infine, si procederà a un'analisi della costruzione del discorso storico, in altre parole un'analisi del come la scelta della voce narrante e l'impostazione della memoria contribuiscono alla narrazione della storia ovvero alla storiografia.

\section{Regina di fiori e di perle di Gabriella Ghermandi e Memorie di una principessa etiope di Martha Nasibù}

Il romanzo di Gabriella Ghermandi ha una struttura simile a quella di una scatola cinese: raccoglie più racconti, contenenti, alcuni di loro altre narrazioni interne di secondo (metadiegesi) e di terzo livello (metametadiegesi). La cornice è molto semplice: si tratta della storia di Mahlet, protagonista del romanzo, prima una bambina che cresce in Etiopia, poi una giovane donna che parte per studiare in Italia. Mahlet bambina ama ascoltare le storie dei grandi. In una delle prime scene del libro la ragazzina promette al vecchio Jacob, uno degli anziani della comunità, di scrivere in futuro la sua storia che egli le racconta. Col tempo Mahlet si scorda della promessa e, anni più tardi, tornata dall'Italia per piangere la morte del vecchio, dovrà ascoltare altri sei racconti seduta nel cortile di una chiesa ${ }^{6}$, per riuscire a ricordarsi della parola data a Jacob. Essi occupano tutta la seconda parte e quasi una metà del romanzo. Tutti, tranne l'ultimo, parlano della guerra italo-etiopica del 1935-1936. La promessa finalmente riemersa dall'oblio - siamo alla fine del libro - Mahlet compra trenta quaderni che riempirà con la storia del vecchio Jacob e con tutte le altre riferitele, che il lettore capisce di aver appena letto leggendo l'ultima metanarrativa frase finale: «Ed è per questo che vi racconto oggi la sua storia. Che poi è anche la mia. Ma pure la vostra» (GG: 299), parole nelle quali la voce della protagonista

${ }^{5}$ Qui Rosner riferisce e ribadisce soprattutto la teoria di David CARR (1986) sulla natura narrativa del vissuto umano, della sua esperienza del mondo, del tempo e della società. Le narrazioni storiche e letterarie vanno quindi considerate come una configurazione e come uno sviluppo di queste esperienze (Rosner, 2006: 91).

${ }^{6}$ Cfr. Proto Pisani (2016: 210) - tipologia dei racconti, introduttivo di Yacob, racconto allegorico tradizionale, apologetico sugli eroi della resistenza, reportage sull'emigrazione contemporanea. 
diventa la voce della scrittrice che di fatto scrive il romanzo che stiamo per finire di leggere.

Si desume che tale intreccio comporti alcune soluzioni narrative piuttosto complesse: si assiste alla pluralità di voci narrative, tra cui quella intradiegeticaomodiegetica della protagonista in parte sovrapposta a quella della scrittrice al livello della diegesi. All'interno di questa cornice narrativa di primo livello troviamo poi situazioni narrative (metadiegesi) di diverso tipo: quella extradiegetica-eterodiegetica dell'anziano vescovo (Storia dello stupido leone con la scimmia), quella intradiegetica-autodiegetica di Abbaba Igirsà Salò che racconta della sua attività come wst arbegnà, ovvero una specie di partigiano della resistenza sotto copertura, poi quella intradiegetica-omodiegetica di Dinke che descrive le gesta di Farisa Alula, il Grande. Segue la narrazione intradiegeticaautodiegetica della «signora della tartaruga» che riferisce le storie raccontatele da sua madre, tra cui una sull'Imperatrice Taytu e una su lei stessa al servizio della guerriera Kebedech Seyoum. In queste narrazioni di terzo livello (metametadiegesi) la voce narrante è rispettivamente extradiegetica-autodiegetica e intradiegetica-omodiegetica. Nell'ultimo racconto inserito si assiste alla narrazione intradiegetica-omodiegetica di Woizero Bekelech all'interno della quale - di nuovo siamo di fronte al terzo livello narrativo (metametadiegesi) - viene inserito il racconto di tipo intradiegetico-omodiegetico del signor Antonio, un anziano italiano che lavorò come interprete dell'amarico durante il conflitto del '35-'36 e nel periodo della successiva colonizzazione dell'Etiopia. La pluralità di voci narranti nonché la varietà delle loro tipologie producono, considerata la loro valenza letteraria, l'effetto di polifonia e di coralità. In chiave di indagine storica invece, ci si potrebbe vedere un materiale per la cosiddetta storia orale, metodo non convenzionale della ricerca storica, di cui scrive Paul Thompson che: «Nel processo di scrivere o di creare la storia - a prescindere se si tratta di libri, mostre museali, radio o film - la storia orale restituisce la posizione centrale alle persone che trasmettono le loro esperienze storiche a mezzo delle proprie parole» $\rangle^{7}$ (THOMPSON, 2010: 283).

Il secondo libro preso in esame Memorie di una principessa etiope di Martha Nasibù è un racconto autobiografico, ma è da considerare anche una testimonianza storica. A distanza di sessant'anni l'autrice raccoglie i ricordi che risalgono agli anni più teneri della sua vita, all'inizio degli anni Trenta o anche a quelli precedenti la sua nascita, e quindi basati sui racconti della madre, arrivando fino all'anno 1946, quando quindicenne Martha insieme al fratello Brahanou lascia il paese natio per conseguire la licenza media superiore a Ginevra.

Il libro è diviso in due parti. La prima rievoca la felice infanzia e la storia della famiglia prima del '35 nonché la figura paterna del grande e stimatissimo degiac Nasibù Zeamanuel, un uomo intelligente e istruito nonché un guerriero

\footnotetext{
7 Trad. - B.K.
} 
carismatico e coraggioso, e un politico abile e moderno, kantibà (sindaco) di Addis Abeba. Nella seconda parte del libro Martha ricorda invece la lunga Odissea cui fu sottoposta la famiglia Nasibù-Babitcheff, tra il 1937 e il 1945 in esilio, spostata di continuo tra Napoli, Tripoli, Vigo di Fassa, Firenze, Rodi, Napoli, campagna aretina, Firenze, Pozzo di Fassa, Roma e Bari, sempre sorvegliata e controllata dal regime mussoliniano e, dopo la sua caduta, abbandonata letteralmente a sé stessa senza un minimo sostegno finanziario, in balia della fame, della povertà e di tutte le insicurezze e tutti i terrori della guerra.

Dal punto di vista della tecnica narrativa adoperata il libro Memorie di una principessa etiope si presenta in maniera molto più semplice rispetto al romanzo di Gabriella Ghermandi. Si tratta della narrazione di tipo intradiegeticoautodiegetico, quindi la voce narrante è una sola e appartiene alla scrittrice e protagonista al contempo ${ }^{8}$ la quale talvolta percepisce e riferisce il mondo circostante dalla prospettiva di una bambina o una ragazzina e talvolta invece la sua voce si fa più riflessiva, ragionata e adulta, traducendo la sua prospettiva storica a distanza dei sessant'anni trascorsi dalle vicende raccontate.

Come si può quindi evincere, in entrambi i libri è di cruciale importanza la memoria che costituisce non solo il nucleo delle narrazioni ma anche la base su cui viene allestita tutta la narrazione. Conseguiranno da tale allestimento tre effetti: una netta valorizzazione del vissuto personale, il soggettivismo della relazione e la precedenza del passato sul presente o sul futuro.

\section{Memoria e postmemoria}

Paul Ricoeur elenca tre attributi della memoria che determinano il suo carattere nettamente personale: la memoria è sempre individuale ovvero i propri ricordi non si possono trasferire ad altri soggetti; nella memoria si colloca il legame primordiale che la coscienza instaura con il passato; e infine è con la memoria che è connessa la consapevolezza bidirezionale - dal passato al futuro e al contrario - del passare del tempo (Ricoeur, 2006: 127). Tutte queste proprietà della memoria si scorgono nei testi esaminati, sia al livello della diegesi sia a quello della metadiegesi e metametadiegesi, basandosi entrambi - in dimensioni diverse in ambedue i casi - su ricordi intimi, aderenti all'esperienza personale del passato e derivanti proprio dal vissuto dei narratori, ricordi proiettanti anche sul futuro in una misura più ampia che quella solamente personale. Inoltre parrebbe risultare fruttuosa per la presente analisi la distinzione aristotelica tra due tipi dei ricordi, tra mnēmè e amnēsis, citata da Ricoeur (2006: 31-33), dove il

\footnotetext{
${ }^{8}$ L'identificazione avviene in base al patto autobiografico. Cfr. Le JEUne (2001: 21-56).
} 
primo elemento, $m n \bar{e} m \bar{e}$, un semplice ricordo, nasce alla stregua di un'emozione e il secondo, amnēsis, è un richiamo del passato che consiste in una ricerca attiva. Sulla base di questa distinzione si costruisce la distinzione tra la memoria e la storia (LoBA, 2004: 296).

Tutta la struttura del romanzo della Ghermandi è impostata sul concetto della memoria e dei ricordi, cosa che si desume in primo luogo dall'accostamento dell'ultima, significativa e già menzionata frase: «E per questo che oggi vi racconto la sua storia. Che poi è anche la mia. Ma pure la vostra» (GG: 299) con le parole iniziali del libro: «Quando ero piccola [...]» (GG: 5). Si costruisce in questa maniera una cornice narrativa e temporale entro la quale si collocano i ricordi della protagonista dall'infanzia al momento in cui essi vengono scritti, i quali, a loro volta, contengono i ricordi degli altri narratori. Le prime parole del romanzo costituiscono il preannuncio dell'intento narrativo (discorso intimo e in prima persona) e un immediato spostamento sul piano delle rimembranze della narratrice, nonché segnano il punto nel passato cui risale la sua memoria: «Quando ero piccola». L'ultima frase svela la doppia identità della narratrice (protagonista-scrittrice), giustificando al contempo la sua necessità di narrare i ricordi. In secondo luogo, lo schema narrativo è allestito sulla promessa data al vecchio Jacob e in seguito scordata dalla protagonista per cui il lavoro della memoria (scordare/ricordare) costituisce l'intelaiatura dell'opera. Infatti, tutti i racconti che occupano la seconda parte del romanzo - si tratta anche qui del lavoro della memoria - nell'economia romanzesca devono aiutare Mahlet a ripescare dalla sua memoria la promessa rimossa. La funzione narrativa di questi racconti funge altresì, od oltretutto, da pretesto per ricordare la storia dell'Etiopia, in particolare quella della guerra del '35-'36. Essi tuttavia non portano all'esito tanto auspicato: Mahlet non riesce a ricordarsi della promessa. Soltanto la vista del vecchio quaderno scolastico che Mahlet da piccola regalò al vecchio Jacob perché ci annotasse la sua storia la condurrà all'esito desiderato. Ghermandi registra il meccanismo operativo della memoria - simile qui al famoso procedimento proustiano - la quale, grazie alle percezioni sensoriali (qui grazie alla vista e al tatto: «Accarezzai la copertina lentamente, fino al bordo interiore [...]» (GG: 292)), si apre («Qualcosa dentro di me si squarciò» (GG: 292)) e restituisce alla coscienza i contenuti rimossi («Certo che era ben nascosta!» (GG: 292)). Infine, anche le narrazioni inserite nel quadro della cornice principale - le quali sono già di per sé, quasi tutti, ricordi dai tempi della guerra italo-etiopica contengono in sé altre micronarrazioni, presentate come frutto della memoria involontaria $(m n \bar{e} m \bar{e})$. Talvolta, in tali casi Ghermandi ricorre alla metafora della vista che oltre al visibile vede altro:

Il mio sguardo si allungò fino ai bracci montuosi che circondavano il Catino del viso di Maria. Bracci senza il folto di alberi della foresta. Solo erba quasi secca, aloe con i fiori rossi e qualche cespuglio di piante spinose. Era uguale 
alla vegetazione di un altro luogo. Degli altipiani del Tigrai, dei pianori del nord.

GG: 29

Oppure che permette di "vedere" il passato: «Ho ancora negli occhi il primo attacco che facemmo alla camionetta con le armi» (GG: 39). Metafora questa che sarebbe da ricollegare alla tradizione di matrice santagostiniana di considerare la memoria come sguardo interiore (RICOEUR, 2006: 127-134).

Il secondo dei libri analizzati con il titolo stesso, Memorie di una principessa etiope, impone una chiave interpretativa, iscrivendosi il testo di Nasibù nella tradizione memorialistica e quindi quella di un genere letterario con alcune determinate caratteristiche, tra cui quella di un chiarissimo intento che sta dietro alla stesura delle memorie. L'autrice lo espone esplicitamente nella Premessa, dove elenca i motivi che la spinsero a scrivere il libro. In primo luogo, si trattava dell'incoraggiamento da parte dei figli consapevoli che la sua opera «avrebbe costituito un legame con il passato della famiglia attraverso l'odissea del loro celebre nonno, il degiac Nasibù Zamanuel» (MN: 21). In secondo luogo «è stato il senso del dovere che l'ha guidata nel riportare alla luce questa memoria, che diversamente sarebbe andata perduta soprattutto per i suoi figli e i suoi nipoti» (MN: 21). E per finire la sua premessa Martha Nasibù scrive: «Un filo d'oro attraversa la mia mente e si dipana nei meandri della memoria per ridare vita ai fatti, anche i più personali, che hanno attraversato la mia esistenza e quella di chi mi è vissuto accanto» (MN: 21). Come si può notare chiaramente le ragioni che portarono Martha Nasibù a scrivere le sue memorie aderiscono molto bene agli attributi della memoria stessa elencati da Ricoeur: carattere individuale, nesso tra coscienza e passato, collegamento tra passato e futuro. Inoltre, i ricordi trascritti e inseriti nelle Memorie di una principessa etiope, visto che si tratta di un testo preceduto da un lavoro di sistemazione e di elaborazione del materiale fornito dalla memoria, sarebbero da collocare nell'ambito di amnēsis, ovvero della memoria che si trasforma nella ricerca e nella rappresentazione della storia. Infatti, alla stesura definitiva del libro contribuì anche lo storico Angelo Del Boca. Nella sua prefazione alle Memorie leggiamo: «Non è stata un'impresa facile, per Martha Nasibù, evocare e riordinare tanti ricordi e poi confrontarli scrupolosamente con gli avvenimenti storici. Ma Martha non si è arresa alle prime difficoltà e ha accettato i miei suggerimenti anche quando comportavano ampi rifacimenti o addirittura nuove stesure del testo. Sono occorsi cinque anni per mettere a punto questo documento [...]» (Del BocA, 2012: 9).

Nei ricordi di Martha Nasibù si possono individuare principalmente due tipi di narrazioni che rispecchiano due diversi procedimenti della memoria. Qualche volta la sua voce cerca di rievocare, riprodurre e descrivere le sue sensazioni, le sue percezioni e la maniera in cui concepiva e capiva il mondo circostante da 
bambina o da adolescente, cioè quando realmente visse i fatti riferiti'. L'autrice riflette anche su questo fenomeno della memoria riconducibile alla nozione di $m n \bar{e} m \bar{e}$ : «Ero piccola allora, ma facendo uno sforzo, grazie all'intuizione, alle sensazioni, agli odori, alle immagini visive, molte cose riaffiorano, dapprima come memoria labile e offuscata, poi, a poco a poco, si rivelano con chiarezza» (MN: 143). Altre volte, invece, riporta i suoi ricordi con molta precisione e chiarezza, affiancandoli con riflessioni e spiegazioni razionali che traducono la consapevolezza nonché le capacità di giudizio acquisite con la distanza temporale. Inoltre, soprattutto per quanto riguarda la prima parte delle sue memorie, ovvero fino alla morte del padre il 16 ottobre del 1936, i suoi ricordi sono sostenuti dai racconti della madre perché naturalmente Martha non poteva conoscere niente del periodo precedente la sua nascita (1931) e forse ricordarsi molto poco del periodo della guerra quando aveva quattro e cinque anni, cosa che lei evidenzia con alcuni frammenti sparsi nel testo di tipo: «Mia madre mi raccontò che $[. .]$.$» .$ Lo conferma anche Angelo Del Boca: «[...] la ricostruzione degli avvenimenti nella prima parte del libro, appare a volte incerta, ed è stata sicuramente integrata con i ricordi della madre di Martha [...]» (Del BocA, 2012: 15). Nella prima parte del libro, proprio per questa sfumatura che caratterizza i ricordi di Martha, e cioè che si tratta del ricordo del trauma vissuto tramandatole dalla madre, dopo l'atroce morte del padre si può, all'avviso di chi scrive, parlare in questo momento del fenomeno della postmemoria. Tale termine, coniato da Marianne Hirsch in relazione all'esperienza dei bambini superstiti dell'olocausto, può essere altresì applicato più generalmente alla memoria della seconda generazione la quale si riferisce agli avvenimenti e alle esperienze traumatiche. La postmemoria caratterizza l'esperienza di chi crebbe in un ambiente dominato dalle narrazioni precedenti la sua nascita (HIRSCH, 2010: 254-255). Per più motivi, tra cui la descrizione dei traumi della guerra italo-etiopica quali l'uso dei gas, le persecuzioni, l'esilio, in relazione poi alla mancanza di consapevolezza di Martha, troppo piccola per capire la realtà, e le successive narrazioni della madre che la influenzano, le Memorie... di Martha Nasibù sono un'opera cui si può senz'altro riferire il termine di postmemoria. Inoltre, come spiega la stessa Hirsch, la postmemoria - rispetto alla memoria - è caratterizzata dalla distanza generazionale nonché da un rapporto intimo e profondo con la storia (HIRSCH, 2010: 252).

A questo punto pare opportuno dare spazio a un'osservazione relativa alla memoria. Ewa Domańska sostiene che essa, definita come discorso di rivendicazione e di insurrezione, come voce di chi è stato privato della voce nel discorso storico tradizionale, praticata come una forma di contro-storia, e in tale accezione preposta da alcuni storici, diventa uno strumento molto utile per analizzare le differenze e le alterità, soprattutto nell'ambito delle nuove scienze umane.

${ }^{9}$ Martha Nasibù nacque nel 1931. Il libro riferisce, oltre al periodo dell'invasione e della colonizzazione italiana 1935-1941, il periodo che si estende dai primi anni Venti fino al 1946. 
La memoria quindi nel nuovo paradigma delle new humanities ha soppiantato la storia come disciplina tradizionale, in quanto quest'ultima si dimostra strumentalizzata dal potere e soggetta alle ideologie legittimanti questo potere, e in quanto viene messa in questione la nozione della verità assoluta e contestata l'oggettività nonché la credibilità della storia. Tuttavia, continua la studiosa, neanche la memoria si sottrae al rischio di essere coinvolta nei meccanismi del potere e dell'ideologizzazione (DomAŃsKA, 2006: 14-16). Nelle narrazioni presenti in entrambi i libri, non abbiamo a che fare con tale processo, partendo le memorie ivi registrate dalle posizioni non dominanti e non prevaricatrici, ma piuttosto rivendicatrici di una voce nel capitolo che la riguarda.

Si passa quindi al rapporto tra memoria e storia nelle opere della Ghermandi e della Nasibù.

\section{Storia, contro-storia}

Tra le varie posizioni degli studiosi - storici e filosofi - dediti all'esame del passato, ovvero all'esame della storia e della storiografia intesa come la trascrizione di questa ricerca, riportate da Katarzyna Rosner nella sua opera Narracja, tożsamość, czas (RosNer, 2006), a questo punto sembra opportuno fare riferimento alle voci narrativistiche, tra cui quella di David Carr, filosofo husserliano, secondo il quale il passato storico è una realtà costruita, non dagli storici che la interpretano, bensì dai suoi attori, da coloro che l'hanno vissuta. La realtà storica è una realtà dell'esperienza umana e in quanto tale è già costruita (CARR, 1986, cit. da RosNer, 2006: 100).

Un simile approccio alla storia e alla storiografia sembra del tutto valido nonché pertinente alla maniera in cui la storia viene riportata e trattata in ambedue i libri analizzati. In entrambi, infatti, la storia raccontata deriva dal vissuto capillare dei narratori, dalla loro diretta esperienza sperimentata sulla propria pelle o dall'esperienza riferita dai più prossimi, vissuta da loro e rielaborata dalla memoria (amnessis). Sono gli attori della storia a narrarla e nello stesso tempo a costruirla come discorso storico per le generazioni a venire. È una storia vista dal basso, nel senso che è colta nel suo svolgersi, spoglia dell'apparato di metodi, ideologie e preconcetti, raccontata - aspetto di primo ordine - non solo dai diretti attori, ma anche da chi è stato emarginato nel discorso della storia tradizionale, dalle voci dei perdenti, colonizzati, esiliati, espulsi.

La regina di fiori e di perle è il primo romanzo in lingua italiana e pubblicato in Italia ${ }^{10}$ che parla della guerra italo-etiopica affrontando i dolorosi temi

${ }^{10}$ Memorie di una principessa etiope è un libro precedente di due anni però non può essere considerato un romanzo. 
dell'invasione italiana quali l'uso dell'iprite, la resistenza degli arbegnà, il combattimento delle squadre femminili ${ }^{11}$, repressioni sterminanti per la popolazione etiope, volute dal generale Rodolfo Graziani dopo l'attentato del 19 febbraio del 1937, come nota Barbara De Vivo, «aspetti più inusuali e meno raccontati della memoria del colonialismo italiano» i quali «aprono uno spazio nel panorama italiano per l'inversione dei discorsi binari che tracciano linee di confine e di demarcazione netta nel processo di ricordo del passato coloniale» (De VIVO, 2013: 125). Sembrano cruciali le domande ch si pone la studiosa: «[...] chi ha avuto e continua ad avere il diritto di parola sul passato coloniale italiani? Come è costruita la memoria culturale di questo passato? Lungo quali assi di potere funziona la memoria? Chi ha il diritto di ricordare? Chi prende la parola? Quali prospettive e quali narrazioni diventano senso comune?» (125-126).

Gabriella Ghermandi per parlare della storia ricorre a una tradizione storiografica molto antica: ricostruisce la storia basandosi sulle vere e proprie testimonianze storiche in forma orale che la protagonista doveva ascoltare al fine di tramandare alle generazioni future la memoria di quei tempi e di quegli avvenimenti. Si ha quindi a che fare con lo stesso intento e lo stesso procedimento assunto dalla storiografia etiope stessa e mi riferisco qui a un'opera intitolata Zekre neger (che si può tradurre come Cosa memorabile) (RUBINKOWSKA-ANIOE, WoŁK-Sore, 2014: 331) scritta da Mahteme Syllasje Uelde Mesk'ele, nel 1949, una delle più importanti opere scritte di storiografia etiope, studiata e analizzata dalle studiose polacche Ewa Wołk-Sore e Hanna Rubinkowska-Anioł, paragonata da loro, per quanto riguarda la tecnica di ricostruire la storia e anche dal punto di vista della finalità di scrivere la storia, all'opera e all'operato di Erodoto. Entrambi si basano sulle fonti orali, testimonianze rese dai testimoni oculari oppure riferite e tramandate oralmente. Entrambi inoltre si richiamano alle esperienze della guerra, considerata da una parte come forza distruttrice dall'altra come momento di mobilizzazione delle forze patriottiche e arena delle gesta eroiche (Rubinkowska-Aniol, Wolk-Sore, 2014: 336). All'identica tecnica di scrivere la storia e alla simile intenzione di farlo assistiamo nel romanzo di Ghermandi.

Il libro di Martha Nasibù, invece, come ribadito da Angelo Del Boca nella Prefazione: «[...] non ha soltanto valenza storica per gli episodi assolutamente inediti che rivela, ma anche il grande pregio di condurci in un mondo del tutto sconosciuto a noi occidentali, quello complesso dell'aristocrazia etiopica degli anni Venti e Trenta, in bilico tra le suggestive eredità del feudalismo e le forti aspirazioni alla modernità» (Del BocA, 2012: 9-10). Infatti, la testimonianza

${ }^{11}$ Ha molta rilevanza l'episodio intitolato Storia della signora della tartaruga, evidenziato da Cristina LOMBARDI-Diope (2011: 305-305), che contiene una scena parallela a una presente ne Il tempo di uccidere, con la differenza che è una giovane guerriera etiope a uccidere il soldato italiano. L'analogia e la differenza risuonano nelle parole concludenti la scena: «Purtroppo è questo il nostro tempo. Dobbiamo combattere» (GG: 228). La scena poi metaforizza la contro-storia di cui è manifestazione il romanzo. 
storica che ci offre la narratrice e scrittrice in persona riguarda entrambe, sia la storia politico-bellica volta alla puntualizzazione dei più rilevanti momenti della guerra italo-etiopica e della successiva colonizzazione, sia la storia socioculturale volta alla presentazione degli usi quotidiani, dei riti religiosi e delle usanze nell'ambiente dell'aristocrazia etiope oppure, nella seconda parte del libro, alla situazione socio-economica e la realtà quotidiana dell'Italia durante la Seconda guerra mondiale. Alla prima vengono dedicati alcuni interi capitoli come 3 ottobre: l'Italia aggredisce l'Etiopia oppure Addis Abeba conquistata, dove la voce narrante diventa concreta, precisa, attenta ai fatti, oggettivante e il testo densamente intrecciato di date e di nomi propri, riportando informazioni e dati che rappresentano, la maggior parte delle volte, la percezione delle cose da parte del cosiddetto Altro, costituendo quindi un contributo alla contro-storia. Leggiamo ad esempio relativamente all'atteggiamento dell'Europa:

Due mesi dopo anche l'Inghilterra in accordo con la Francia e altri paesi europei produttori di armi, sospese la vendita di armi all'Italia e all'Etiopia, che si trovò così in situazione di grave inferiorità, dal momento che l'Italia produceva autonomamente il materiale bellico di cui aveva bisogno.

MN: 111

Tra questi fatti, date ed elementi fattuali vi sono inserite non poche impressioni, riflessioni o suggestioni dell'autrice stessa, sempre tuttavia prodotte a posteriori rispetto ai fatti raccontati, essendo Martha troppo piccola in quel periodo per poter pensare le frasi come la seguente: «[...] la volontà di Dio doveva compiersi e la storia doveva trovare il suo spazio per scrivere la sorte dei popoli e delle nazioni» (MN: 114). Riflessione questa che non solo lascia trapelare una profonda fede in Dio, sottintesa nel testo più volte, e prisma attraverso cui vedere tutti fatti accaduti, ma richiama anche alla mente la concezione narrativistica della storia di Carr precedentemente citata: la storia si produce per mezzo dell'esperienza dal vissuto dei suoi attori. Per gli aspetti socio-culturali della storia, anch'essi almeno nella prima parte del libro forniti dalla memoria della madre, Atzede, la narrazione di Martha rimane spesso precisa:

La festa di Maskal è la più pittoresca ricorrenza religiosa, durante la quale si commemora il ritrovamento della Croce di Cristo da parte di sant'Elena, madre dell'Imperatore Costantino il Grande, nel IV secolo d.C. Il Sacro Legno è simbolo della redenzione, riferimento essenziale per i cristiani d'Etiopia.

Talvolta però diventa più suggestiva, incantata, soggetta alle impressioni e alle percezioni: «Il giubileo aveva contagiato grandi e piccoli, grida di gioia echeggiavano nell'oceano di colori, bandiere e festoni, e la folla danzava facendo 
volteggiare in aria i candidi sciamma» (MN: 77). Spesso la voce narrante rimane proprio infantile, ricca di emozioni e sensazioni, quella di Martha bambina.

\section{Concludendo}

Le narrazioni di entrambe le scrittrici, con le voci prevalentemente intradiegetiche e omo- o autodiegetiche, quindi personali, intime, basate sul vissuto e sull'esperienza proprie oppure quelle dei genitori, evidenziano l'importanza della memoria o della postmemoria, nella costruzione della coscienza storica e dell'identità, dal momento, come sostiene M. Loba: «Il soggetto ha bisogno della narrazione per rappresentare la sua memoria e per conservare la sua identità» (LOBA, 2004: 297). In più, nella storia che si narra, quindi attraverso la memoria degli attori e dei testimoni, la voce appartiene alle donne, agli etiopi e ai bambini, tutte istanze subalterne - dal punto di vista di genere, razza e posizione sociale - dei sistemi dominanti della nostra cultura (occidentale) patriarcale, coloniale e androcentrica. Diventano così soggetto del discorso storico coloro che ne hanno sempre costituito solo l'oggetto imponendo una loro voce, una loro verità, nonché contribuendo con ciò a un discorso storico plurale e polifonico.

\section{Bibliografia}

CARr, David, 1986: Time, Narrative and History. Bloomington, University Indiana Press.

Del Boca, Angelo, 2012: "Prefazione". In: Martha Nasibù: Memorie di una principessa etiope. Vicenza, Biblioteca Editori Associati di Tascabili, pp. 5-18.

De VIvo, Barbara, 2013: "Alla ricerca della memoria perduta. Contro-memorie della colonizzazione italiana in Etiopia nel romanzo Regina di fiori e di perle di Gabriella Ghermandi". In: Franca Sinopoli, ed.: Postcoloniale italiano. Tra letteratura e storia. Novalogos, Aprilia, pp. $120-146$.

DomańsKa, Ewa, 2006: Historie niekonwencjonalne. Refleksja o przeszłości w nowej humanistyce. Poznań, Wydawnictwo Poznańskie.

Foucault, Michel, 2012 : "Il faut défendre la société ». Cours au Collège de France (1975-1976),

$<$ https://monoskop.org/images/9/99/Foucault_Michel_Il_faut_defendre_la_societe.pdf $>$. [Data dell'ultima consultazione: 03.07.2019].

Ghermandi, Gabriella, 2011: Regina di fiori e di perle. Roma, Donzelli Editore.

Hirsch, Marianne, 2010: „Żałoba i postpamięć”. In: Ewa Domańska, red.: Teorie wiedzy o przeszłości na tle wspótczesnej humanistyki. Poznań, Wydawnictwo Poznańskie, pp. 247280. 
Le Jeune, Philippe, 2001: Wariacje na temat pewnego paktu. O Autobiografii. Kraków, Universitas.

LobA, Mirosław, 2004: „Pamięć i narracja”. Scripta Neophilologica Posnaniensia, nr 6, pp. 293 298.

LOMBARDi-Diopp, Cristina, 2011: "Postfazione". In: Gabriella GHERmandi: Regina di fiori e di perle. Roma, Donzelli Editore, pp. 305-313.

NAsIBÙ, Martha, 2012: Memorie di una principessa etiope. Vicenza, Biblioteca Editori Associati di Tascabili.

Proto Pisani, Anna, 2016 : «Une écriture à la lisière des genres : Regina di fiori e di perle de Gabriella Ghermandi». In: Olivier Favier, Anna Proto Pisani, Paola RanZini, éd.: Les littératures de la Corne de l'Afrique. Paris, Karthala, Regards croisés, pp. 207-237.

Ricoeur, Paul, 2006: Pamięć, historia, zapomnienie. Kraków, Universitas.

Rosner, Katarzyna, 2006: Narracja, tożsamość, czas. Kraków, Universitas.

Rubinkowska-Aniol, Hanna, WoŁk-Sore, Ewa, 2014: „Etiopski intelektualista i jego dzieło pomiędzy oraturą a literaturą". In: Iwona Kraska-Szlenk, Beata Wójtowicz, eds.: Current research in African studies: papers in honour of Mwalimu Dr. Eugeniusz Rzewuski. Warszawa, Dom Wydawniczy Elipsa, pp. 331-346.

Thompson, Paul, 2010: „Głos przeszłości. Historia mówiona”. In: Ewa Domańska, red.: Teorie wiedzy o przeszłości na tle współczesnej humanistyki. Poznań, Wydawnictwo Poznańskie, pp. 281-294.

\section{Nota biobibliografica}

Barbara Kornacka è professore associato presso il Dipartimento di Lingue e Lettere Romanze dell'Università Adam Mickiewicz di Poznań in Polonia. È specializzata nella letteratura italiana contemporanea. Tra i suoi campi di interesse e di ricerca vi sono: la letteratura dei «giovani scrittori» di fine secolo, la scrittura delle donne, la letteratura italiana postcoloniale, temi cui ha dedicato due libri e diversi articoli. Con il libro del 2013 Ucho, oko, ciało. O prozie ,mtodych pisarzy” lat osiemdziesiatych i dziewięćdziesiatych we Włoszech (Orecchio, occhio, corpo. Sulla narrativa dei ,giovani scrittori" degli anni ottanta e novanta in Italia) ha vinto il Premio Flaiano di Italianistica 2014.

kornacka@amu.edu.pl 